\title{
The Clinicopathological and Prognostic Significance of Nrf2 and Keap1 Expression in Hepatocellular Carcinoma
}

\author{
Kiryang Lee ${ }^{1}\left(\mathbb{D}\right.$, Seunghye Kim ${ }^{1}\left(\mathbb{D}\right.$, Yangkyu Lee $^{2}{ }^{(\mathbb{D}}$, Hyejung Lee $^{1}$, Youngeun Lee $^{1}{ }^{(\mathbb{D}}$, \\ Hyunjin Park ${ }^{3}$, Ji Hae Nahm ${ }^{3}$, Soomin Ahn ${ }^{4}$, Su Jong Yu ${ }^{5}$, Kyoungbun Lee ${ }^{1}$ and \\ Haeryoung Kim 1,*(D) \\ 1 Department of Pathology, Seoul National University Hospital, Seoul National University College of \\ Medicine, Seoul 03080, Korea; pathologic2017@gmail.com (K.L.); pit_a_pat@snu.ac.kr (S.K.); \\ kardia0622@gmail.com (H.L.); nayye@hanmail.net (Y.L.); azirang@gmail.com (K.L.) \\ 2 Department of Pathology, Seoul National University Bundang Hospital, Seoul National University College \\ of Medicine, Seongnam 13620, Korea; gyulgyuli@naver.com \\ 3 Department of Pathology, Gangnam Severance Hospital, Yonsei University College of Medicine, \\ Seoul 06273, Korea; umberto0828@hotmail.co.kr (H.P.); nam2169@yuhs.ac (J.H.N.) \\ 4 Department of Pathology and Translational Genomics, Samsung Medical Center, Sungkyunkwan University \\ School of Medicine, Seoul 06351, Korea; suminy317@gmail.com \\ 5 Department of Internal Medicine and Liver Research Institute, Seoul National University College of \\ Medicine; Biomedical Research Institute, Center for Medical Innovation, Seoul National University Hospital, \\ Seoul 03080, Korea; ydoctor2@hanmail.net \\ * Correspondence: haeryoung.kim@snu.ac.kr; Tel.: +82-(2)-740-8322
}

Received: 16 June 2020; Accepted: 28 July 2020; Published: 31 July 2020

\begin{abstract}
Nuclear factor E2-related factor2 (Nrf2) activation is associated with both cytoprotective effects and malignant behavior of cancer cells. This study aimed to evaluate the clinicopathological implications of the expression of Nrf2, pNrf2, and its regulator Keap1 in human hepatocellular carcinomas (HCCs). Tissue microarrays consisting of 285 surgically resected HCCs were immunohistochemically stained with pNrf2, Nrf2, Keap1, stemness-related markers (keratin 19 (K19), epithelial cell adhesion molecule (EpCAM)), carbonic anhydrase IX (CAIX), epithelial-mesenchymal transition (EMT)-related markers (ezrin, uPAR, E-cadherin), and p53, and the results were correlated with the clinicopathological features. pNrf2 expression was significantly associated with increased proliferative activity, as well as EpCAM, ezrin, p53, and CAIX expression and E-cadherin loss $(p<0.05$, all). Strong cytoplasmic Nrf2 expression was associated with CAIX and ezrin expression $(p<0.05$, both). Keap1 was associated with increased proliferative activity, portal vein invasion, EMT-related markers, and p53 expression in CAIX-negative HCCs $(p<0.05$, all). Both pNrf2 and cytoplasmic Nrf2 expression were associated with decreased overall survival ( $p<0.05$, both), and cytoplasmic Nrf2 expression was an independent predictor of decreased overall survival on multivariate analysis (hazard ratio 4.15, $p<0.001$ ). Both $\mathrm{pNrf} 2$ and cytoplasmic Nrf2 expression were associated with poor survival and aggressive behavior of HCC. In addition, Keap1 expression was also associated with aggressive HCC behavior in CAIX-negative HCCs, suggesting that Keap1 expression should be interpreted in the context of hypoxia status.
\end{abstract}

Keywords: Hepatocellular carcinoma; Nrf2; Keap1; prognosis

\section{Introduction}

Hepatocellular carcinoma (HCC) is the fourth leading cause of cancer-related mortality worldwide [1]. The genomic landscape of HCC was unraveled during the past decade, with the 
most common genetic alterations being mutations in TP53, CTNNB1, and TERT promoter genes [2-4]; following the identification of distinct morphological subtypes of HCCs that partly correlate with the molecular features, there was increasing interest in the heterogeneity of HCC in recent years [5]. This heterogeneity has important clinical implications, as understanding the morphomolecular features of the different subtypes of HCC will lead to increased treatment options and provide background for drug development.

Of the less frequent molecular alterations of HCC, somatic mutations in NFE2L2 and KEAP1 are observed in 3-6\% and 2-8\% of HCCs, respectively $[4,6]$. Activating NFE2L2 mutations and inactivating KEAP1 mutations were demonstrated to play important roles in tumor progression by preventing the degradation of nuclear factor E2-related factor2 (Nrf2), and the Nrf2/Keap1 pathway recently attracted attention as a potential therapeutic target for $\operatorname{HCC}[3,7,8]$. Under physiological conditions, Nrf2 has cytoprotective functions; oxidative stress induces the phosphorylation of Nrf2, and phospho-Nrf2 (pNrf2) is subsequently released from Keap1 to enter the nucleus, bind to antioxidant response elements, and ultimately trigger the expression of cytoprotective genes $[6,9,10]$. However, although this cytoprotective mechanism initially prevents carcinogenesis, the overexpression of $\mathrm{Nrf2}$ in cancer cells may result in protection of cancer cells from oxidative stress, enhancement of cancer cell proliferation and survival, and resistance to therapy [8,11-14].

Although the prognostic and clinical implication of Nrf2 and Keap1 protein expression was recently demonstrated in various malignant neoplasms, previous studies reported conflicting results and there are limited data on HCC [15-22]. In this study, we evaluated the prognostic significance and the clinicopathological features of HCCs expressing Nrf2, pNrf2, and Keap1, and we correlated the findings with the expression status of markers associated with hypoxia (carbonic anhydrase IX; CAIX), stemness (keratin 19; K19, epithelial cell adhesion molecule; EpCAM) and epithelial-mesenchymal transition (EMT) (ezrin, urokinase-type plasminogen activator receptor (uPAR) and E-cadherin).

\section{Results}

The clinicopathological features of the 285 HCC cases are summarized in Table 1 . The most common etiology was hepatitis B virus (HBV) infection (203/285, 71.2\%). Multiplicity was present in 49/285 (17.2\%) cases, and 205/285 (72.0\%) cases were poorly differentiated (Edmondson-Steiner grade 3 or 4). Microvascular and portal vein invasion was seen in 113/285 (39.6\%) and 19/285 (6.7\%) cases, respectively. After a follow up period of 66.5 months (range, 0-163 months), 161/285 (56.5\%) cases recurred and 54/285 (18.9\%) patients died.

Table 1. Summary of the clinicopathological features of hepatocellular carcinomas $(n=285)$.

\begin{tabular}{cc}
\hline Parameters & Frequency (\%) or Median (Range) \\
\hline Sex, $n(\%)$ & $216(75.8)$ \\
\hline Male & $69(24.2)$ \\
\hline Female & $63(29-87)$ \\
\hline Age at operation, median (range, years) & $202(70.9)$ \\
\hline Etiology, $n(\%)$ & $25(8.8)$ \\
\hline Hepatitis B virus (HBV) & $1(0.3)$ \\
\hline Hepatitis C virus (HCV) & $14(4.9)$ \\
\hline HBV + HCV & $12(4.2)$ \\
\hline Alcohol & $31(10.9)$ \\
\hline Non-alcoholic fatty liver disease &
\end{tabular}


Table 1. Cont.

\begin{tabular}{|c|c|}
\hline Parameters & Frequency (\%) or Median (Range) \\
\hline Tumor size, median (range, $\mathrm{cm}$ ) & $3.2(0.9-17)$ \\
\hline Multiplicity present, $n(\%)$ & $49(17.2)$ \\
\hline \multicolumn{2}{|l|}{ Edmondson-Steiner grade, $n(\%)$} \\
\hline Grade I & $3(1.0)$ \\
\hline Grade II & $77(27.0)$ \\
\hline Grade III & $171(60.0)$ \\
\hline Grade IV & $34(12.0)$ \\
\hline Microvascular invasion present, $n(\%)$ & $113(39.6)$ \\
\hline Portal vein invasion present, $n(\%)$ & $19(6.7)$ \\
\hline \multicolumn{2}{|l|}{ Pathologic T category (AJCC 8th edition), $n(\%)$} \\
\hline pT1a & $37(13.0)$ \\
\hline pT1b & $101(35.4)$ \\
\hline pT2 & $112(39.3)$ \\
\hline pT3 & $11(3.9)$ \\
\hline pT4 & $24(8.4)$ \\
\hline Extrahepatic metastasis present, $n(\%)$ & $55(19.3)$ \\
\hline Cirrhosis in background liver present, $n(\%)$ & $152(533.3)$ \\
\hline Recurrence on follow-up present, $n(\%)$ & $161(56.5)$ \\
\hline \multicolumn{2}{|l|}{ Status at last follow-up, $n(\%)$} \\
\hline Alive & $128(44.9)$ \\
\hline Deceased of disease & $44(15.4)$ \\
\hline Deceased of other cause & $10(3.5)$ \\
\hline Follow up loss & $103(36.2)$ \\
\hline Overall survival, median (range, months) & $66.5(0-163)$ \\
\hline Disease-free survival, median (range, months) & $32.5(0-163)$ \\
\hline
\end{tabular}

AJCC, American Joint Committee on Cancer.

\section{1. pNrf2 Expression in HCCs and Clinicopathological Correlation}

The immunohistochemical stain results and clinicopathological data are demonstrated in Figure 1 and Table 2. pNrf2 expression was observed in 73/285 (25.6\%) cases, and all positive cases demonstrated strong nuclear labeling. High pNrf2 expression was associated with significantly higher proliferative activity (mitotic index: $17.4 \pm 20.0 \%$ vs. $10.1 \pm 13.4 \%$, respectively, $p<0.001$; Ki-67 labeling index $(12.9 \pm 12.2$ vs. $5.1 \pm 7.5 / 10$ high-power field (HPF), respectively, $p<0.001)$ compared with pNrf2-low HCCs. pNrf2-high HCCs demonstrated significantly more frequent expression of EpCAM (41/73 $(56.2 \%)$ vs. $84 / 212(39.6 \%)$, respectively, $p=0.020)$, CAIX $(27 / 72(37.5 \%)$ vs. $48 / 205(23.4 \%)$, respectively, $p=0.030)$, ezrin $(43 / 72(59.7 \%)$ vs. 78/212 (36.8\%), respectively, $p=0.001)$, E-cadherin loss $(56 / 73(76.7 \%)$ vs. $128 / 212(60.4 \%)$, respectively, $p=0.015)$, and p53 overexpression $(26 / 70(37.1 \%)$ vs. $40 / 195(20.5 \%)$, respectively, $p=0.009$ ), compared with pNrf2-low HCCs (Figure 2). K19 expression was also slightly more frequent in pNrf2-high HCCs; however, the difference was not statistically significant. pNrf2-high status was significantly associated with decreased overall survival (OS) on univariate analysis (hazard ratio (HR), 1.89: 95\% confidence interval $(\mathrm{CI}), 1.04-3.46 ; p=0.038)$ (Table 3, Figure 3). There was no significant difference in disease-free survival (DFS) according to pNrf2 status. However, analysis of an 
independent cohort of 293 surgically resected HCCs also revealed a significant association between pNrf2 expression and decreased OS ( $p=0.001)$ and DFS ( $p=0.029)$ (Figure S1).

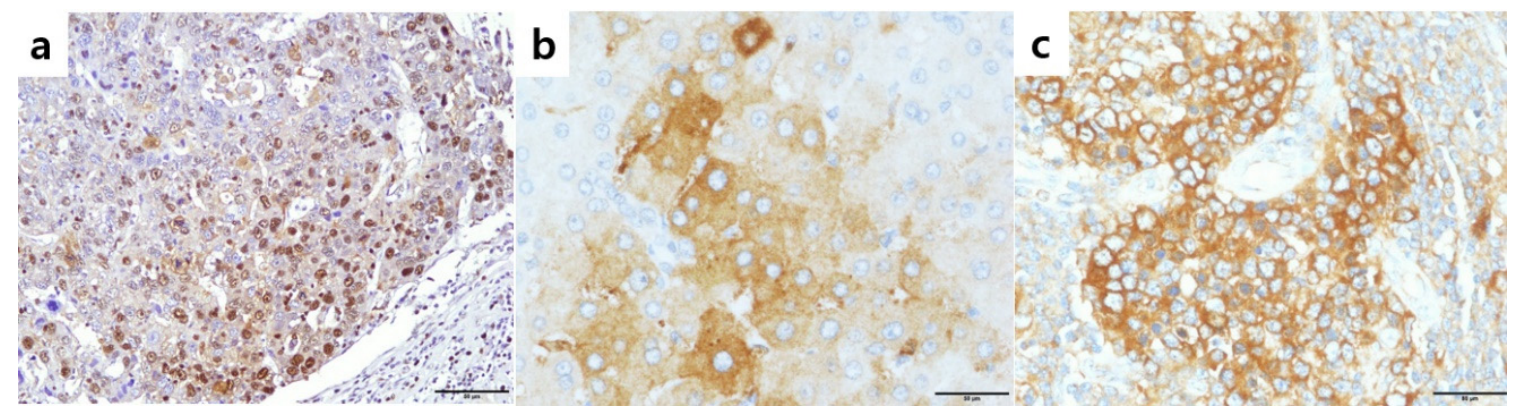

Figure 1. Expression of phosphorylated nuclear factor E2-related factor2 (pNrf2), Nrf2, and Keap1 in hepatocellular carcinomas. (a) Nuclear pNrf2 expression in 63\% of tumor cells; (b) high cytoplasmic Nrf2 expression in tumor cells; (c) high cytoplasmic Keap1 expression in tumor cells. Original magnification $\times 200(\mathbf{a}), \times 400(\mathbf{b}, \mathbf{c})$.

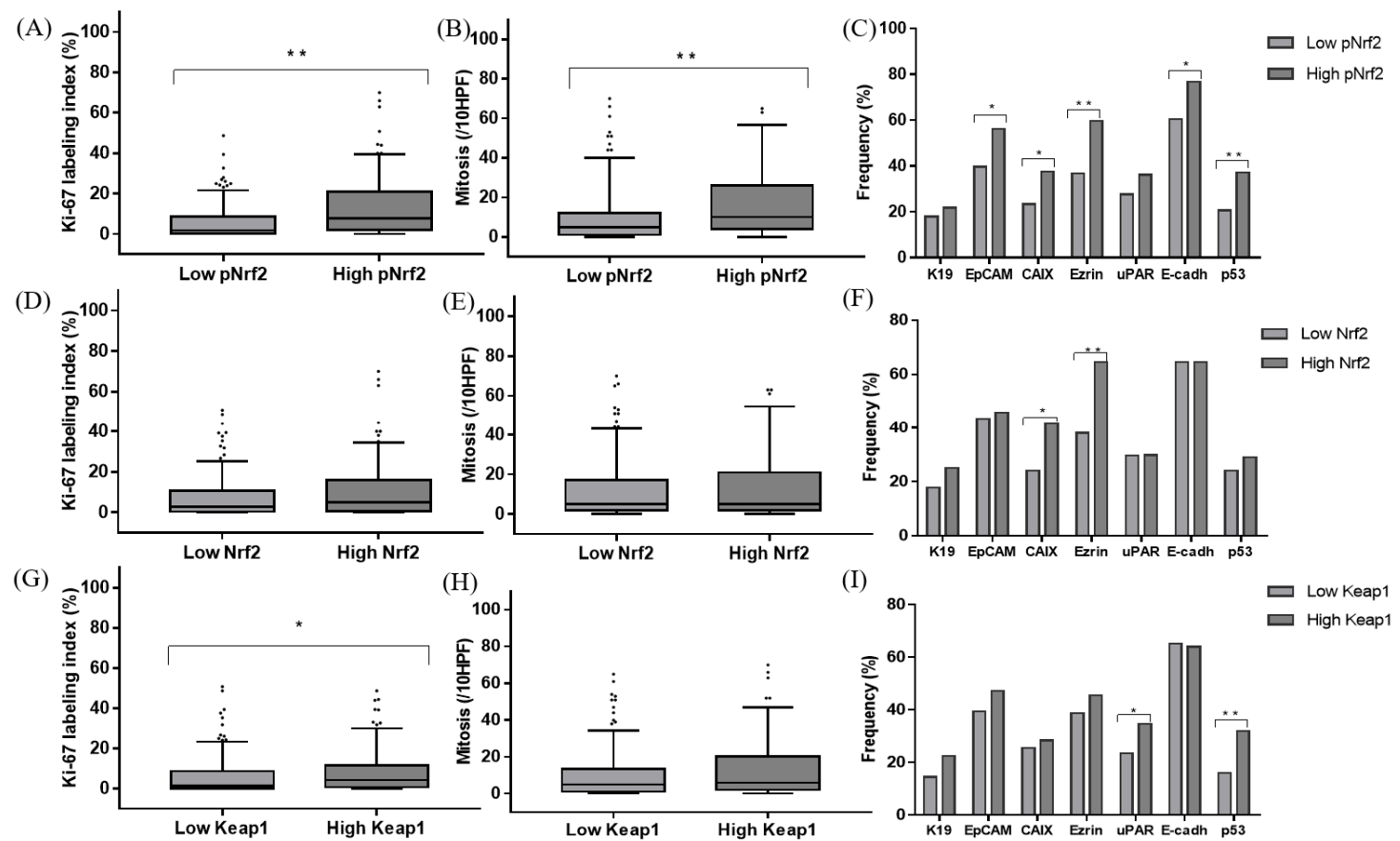

Figure 2. Comparison of the Ki-67 labeling indices $(\mathbf{A}, \mathbf{D}, \mathbf{G})$, mitotic indices $(\mathbf{B}, \mathbf{E}, \mathbf{H})$, and immunohistochemical characteristics (C,F,I) of hepatocellular carcinomas according to $\mathrm{pNrf} 2(\mathbf{A}-\mathbf{C})$, Nrf2 (D-F), and Keap1 (G-I) expression status; ${ }^{*} p<0.05,{ }^{* *} p<0.01 ;$ HPF: high-power field. 
Table 2. Summary of the clinicopathological and immunohistochemical features of hepatocellular carcinomas according to pNrf2, Nrf2 and Keap1 status ( $n=285), n(\%)$.

\begin{tabular}{|c|c|c|c|c|c|c|c|c|c|}
\hline \multirow{2}{*}{ Parameter } & \multicolumn{2}{|c|}{ pNrf2 } & \multirow{2}{*}{$p$-Value } & \multicolumn{2}{|c|}{ Nrf2 } & \multirow{2}{*}{$p$-Value } & \multicolumn{2}{|c|}{ Keap1 } & \multirow{2}{*}{$p$-Value } \\
\hline & Low pNrf2 & High pNrf2 & & Low Nrf2 & High Nrf2 & & Low Keap1 & High Keap1 & \\
\hline Frequency & $212(74.3)$ & $73(25.6)$ & - & $237(83.2)$ & $48(16.8)$ & - & $124(43.5)$ & $161(56.5)$ & - \\
\hline Sex (male/female) & $159(75.0) / 53(25.0)$ & $57(78.1) / 16(21.9)$ & 0.638 & 179 (75.5)/58 (24.5) & $37(77.1) / 11(22.9)$ & 1.000 & $94(75.8) / 30(24.2)$ & $122(75.8) / 39(24.2)$ & 1.000 \\
\hline Age $\geq 60$ years & $95(44.8)$ & $28(38.4)$ & 0.411 & $100(42.2)$ & $23(47.9)$ & 0.542 & $55(44.7)$ & $68(42.2)$ & 0.809 \\
\hline HBV-related etiology & $150(70.8)$ & $53(72.6)$ & 0.881 & $167(70.5)$ & $36(75.0)$ & 0.602 & $86(69.4)$ & $117(72.7)$ & 0.598 \\
\hline Size $(\mathrm{cm}$, mean $\pm \mathrm{SD})$ & $4.2 \pm 3.0$ & $4.0 \pm 2.4$ & 0.274 & $4.4 \pm 2.9$ & $3.1 \pm 1.8$ & 0.005 & $4.4 \pm 3.1$ & $3.9 \pm 2.5$ & 0.105 \\
\hline Multiplicity & $37(17.5)$ & $12(16.4)$ & 1.000 & $41(17.3)$ & $8(16.7)$ & 1.000 & $23(18.5)$ & $26(16.1)$ & 0.636 \\
\hline E-S grade III and IV & $155(73.1)$ & $50(68.5)$ & 0.454 & $173(73.0)$ & $32(66.7)$ & 0.382 & $92(74.2)$ & $113(70.2)$ & 0.507 \\
\hline Microvascular invasion & $78(36.8)$ & $35(47.9)$ & 0.098 & $96(40.5)$ & $17(35.4)$ & 0.628 & $47(37.9)$ & $66(41.0)$ & 0.627 \\
\hline Portal vein invasion & $12(5.7)$ & $7(9.6)$ & 0.278 & $17(7.2)$ & $2(4.2)$ & 0.750 & $12(9.7)$ & $7(4.3)$ & 0.094 \\
\hline pT category & & & 0.146 & & & 0.323 & & & 0.166 \\
\hline pT1a & $31(14.6)$ & $6(8.2)$ & & $33(13.9)$ & $4(8.3)$ & & $18(14.5)$ & $19(11.8)$ & \\
\hline pT1b & $75(35.4)$ & $26(35.6)$ & & $83(35.0)$ & $18(37.5)$ & & $46(37.1)$ & $55(34.2)$ & \\
\hline pT2 & $76(35.8)$ & $36(49.3)$ & & 89 (37.6) & $23(47.9)$ & & $41(33.1)$ & $71(44.1)$ & \\
\hline pT3 & $10(4.7)$ & $1(1.4)$ & & $11(4.6)$ & $0(0)$ & & $4(3.2)$ & $7(4.3)$ & \\
\hline pT4 & $20(9.4)$ & $4(5.5)$ & & $21(8.9)$ & $3(6.3)$ & & $15(12.1)$ & $9(5.6)$ & \\
\hline Recurrence & $118(55.7)$ & $43(58.9)$ & 0.784 & $133(56.1)$ & $28(58.3)$ & 0.748 & $64(51.6)$ & $97(60.2)$ & 0.183 \\
\hline Extrahepatic metastasis & $37(17.6)$ & $18(24.7)$ & 0.229 & $50(21.2)$ & $5(9.1)$ & 0.109 & $24(19.5)$ & $31(19.4)$ & 1.000 \\
\hline Underlying cirrhosis & $108(52.9)$ & $44(60.3)$ & 0.338 & $125(54.1)$ & $27(58.7)$ & 0.628 & $65(55.1)$ & $87(54.7)$ & 1.000 \\
\hline K19 expression & $38(17.9)$ & $16(21.9)$ & 0.490 & $42(17.7)$ & $12(25.0)$ & 0.233 & $18(14.5)$ & $36(22.4)$ & 0.127 \\
\hline EpCAM expression & $84(39.6)$ & $41(56.2)$ & 0.020 & 103 (43.5) & $22(45.8)$ & 0.873 & $49(39.5)$ & $76(47.2)$ & 0.229 \\
\hline CAIX expression & $48(23.4)$ & $27(37.5)$ & 0.030 & $55(24.0)$ & $20(41.7)$ & 0.019 & $31(25.4)$ & $44(28.4)$ & 0.589 \\
\hline Ezrin expression & $78(36.8)$ & $43(59.7)$ & 0.001 & $90(38.1)$ & $31(64.6)$ & 0.001 & $48(38.7)$ & $73(45.6)$ & 0.277 \\
\hline uPAR expression & $58(27.5)$ & $26(36.1)$ & 0.180 & $70(29.7)$ & $14(29.8)$ & 1.000 & $29(23.4)$ & $55(34.6)$ & 0.049 \\
\hline E-cadherin loss & $128(60.4)$ & $56(76.7)$ & 0.015 & $153(64.6)$ & $31(64.6)$ & 1.000 & $81(65.3)$ & $103(64.0)$ & 0.901 \\
\hline p53 overexpression & $40(20.5)$ & $26(37.1)$ & 0.009 & $53(24.1)$ & $13(28.9)$ & 0.570 & $19(16.1)$ & $47(32.0)$ & 0.004 \\
\hline Mitotic index (/10 HPF, mean $\pm \mathrm{SD})$ & $10.1 \pm 13.4$ & $17.4 \pm 20.0$ & $<0.001$ & $11.9 \pm 15.6$ & $12.2 \pm 16.1$ & 0.623 & $10.5 \pm 13.6$ & $13.1 \pm 16.9$ & 0.056 \\
\hline Ki-67 labeling index $(\%$, mean $\pm \mathrm{SD})$ & $5.1 \pm 7.5$ & $12.9 \pm 12.2$ & $<0.001$ & $7.2 \pm 9.6$ & $6.7 \pm 9.6$ & 0.935 & $5.7 \pm 8.5$ & $8.1 \pm 10.2$ & 0.035 \\
\hline
\end{tabular}

HR: hazard ratio, CI: confidence interval, HBV: hepatitis B virus, E-S grade: Edmondson-Steiner grade, K19: keratin 19, EpCAM: epithelial cell adhesion molecule, CAIX: carbonic anhydrase IX; uPAR: urokinase-type plasminogen activator receptor, HPF: high-power field. 
Table 3. Summary of the survival analysis results $(n=285)$.

\begin{tabular}{|c|c|c|c|c|c|c|c|c|}
\hline \multirow{3}{*}{$\begin{array}{l}\text { Parameter } \\
\text { Parameters }\end{array}$} & \multicolumn{4}{|c|}{ Overall Survival } & \multicolumn{4}{|c|}{ Disease-Free Survival } \\
\hline & \multicolumn{2}{|c|}{ Univariate Analysis } & \multicolumn{2}{|c|}{ Multivariate Analysis } & \multicolumn{2}{|c|}{ Univariate Analysis } & \multicolumn{2}{|c|}{ Multivariate Analysis } \\
\hline & $\operatorname{HR}(95 \% \mathrm{CI})$ & $p$-Value & $\operatorname{HR}(95 \% \mathrm{CI})$ & $p$-Value & $\mathrm{HR}(95 \% \mathrm{CI})$ & $p$-Value & $\operatorname{HR}(95 \% \mathrm{CI})$ & $p$-Value \\
\hline Sex (male/female) & $0.742(0.345-1.597)$ & 0.446 & - & - & $0.702(0.480-1.029)$ & 0.069 & - & - \\
\hline Age $(\geq 60 /<60$ years $)$ & $0.960(0.522-1.765)$ & 0.894 & - & - & $0.848(0.621-1.157)$ & 0.299 & - & - \\
\hline Underlying HBV & $1.160(0.586-2.296)$ & 0.671 & - & - & $1.129(0.801-1.590)$ & 0.488 & - & - \\
\hline Size $(\geq 5 \mathrm{~cm} /<5 \mathrm{~cm})$ & $2.400(1.315-4.382)$ & 0.004 & $1.298(0.650-2.594)$ & 0.460 & $1.418(1.012-1.988)$ & 0.043 & $1.085(0.765-1.540)$ & 0.647 \\
\hline Multiplicity & $2.812(1.488-5.312)$ & 0.001 & $2.156(1.093-4.253)$ & 0.027 & $1.991(1.375-2.884)$ & $<0.001$ & $1.487(1.015-2.180)$ & 0.042 \\
\hline E-S grade III and IV & $0.951(0.497-1.818)$ & 0.879 & - & - & $1.095(0.778-1.542)$ & 0.601 & - & - \\
\hline Microvascular invasion & $1.477(0.813-2.682)$ & 0.200 & - & - & $1.308(0.962-1.779)$ & 0.087 & - & - \\
\hline Portal vein invasion & $3.289(1.386-7.804)$ & 0.007 & $2.435(0.980-6.052)$ & 0.055 & $1.573(0.853-2.903)$ & 0.147 & - & - \\
\hline Extrahepatic metastasis & $4.458(2.445-8.130)$ & $<0.001$ & $3.256(1.742-6.083)$ & $<0.001$ & 4.799 (3.411-6.753) & $<0.001$ & $4.475(3.152-6.354)$ & $<0.001$ \\
\hline Underlying cirrhosis & $1.282(0.702-2.340)$ & 0.419 & - & - & $1.031(0.758-1.404)$ & 0.844 & - & - \\
\hline K19 & $1.860(0.957-3.614)$ & 0.067 & - & - & $1.209(0.826-1.771)$ & 0.329 & - & - \\
\hline EpCAM & 1.727 (0.951-3.137) & 0.073 & - & - & $0.889(0.613-1.292)$ & 0.538 & - & - \\
\hline CAIX & $1.854(1.015-3.386)$ & 0.044 & $1.402(0.738-2.664)$ & 0.302 & $1.043(0.743-1.464)$ & 0.809 & - & - \\
\hline Ezrin & $3.160(1.674-5.966)$ & $<0.001$ & $2.893(1.527-5.483)$ & 0.001 & $1.090(0.766-1.552)$ & 0.632 & - & - \\
\hline uPAR & $1.492(0.812-2.743)$ & 0.198 & & & $1.058(0.756-1.480)$ & 0.742 & & \\
\hline E-cadherin loss & $1.037(0.556-1.936)$ & 0.909 & & & $0.853(0.622-1.169)$ & 0.323 & & \\
\hline p53 overexpression & $1.777(0.961-3.288)$ & 0.067 & - & - & $1.219(0.854-1.740)$ & 0.276 & - & - \\
\hline Nrf2 & $3.329(1.738-6.374)$ & $<0.001$ & $4.151(2.025-8.508)$ & $<0.001$ & $1.315(0.879-1.968)$ & 0.183 & - & - \\
\hline pNrf2 & $1.894(1.037-3.458)$ & 0.038 & $1.181(0.950-3.450)$ & 0.071 & $0.933(0.661-1.317)$ & 0.693 & - & - \\
\hline Keap1 & $1.061(0.582-1.937)$ & 0.846 & - & - & $1.290(0.944-1.762)$ & 0.109 & - & - \\
\hline
\end{tabular}

HBV: hepatitis B virus, HR: hazard ratio, CI: confidence interval, SD: standard deviation, E-S grade: Edmondson-Steiner grade, K19: keratin 19, EpCAM: epithelial cell adhesion molecule,

CAIX: carbonic anhydrase IX, uPAR: urokinase-type plasminogen activator receptor, HPF: high-power field. 
(A)

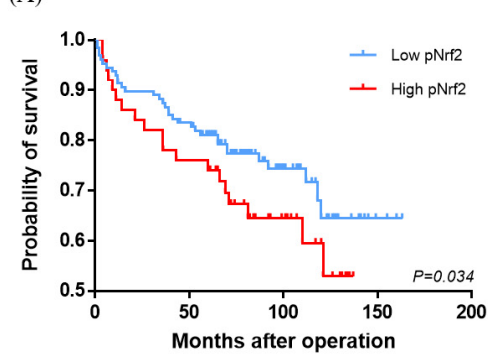

(D)

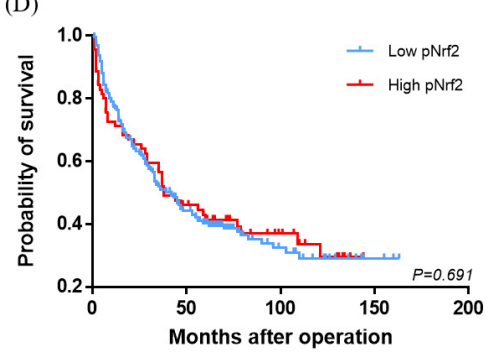

(B)

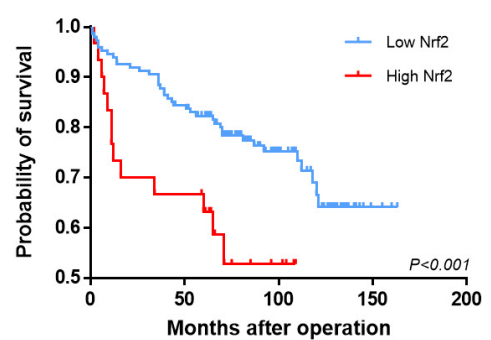

(E)

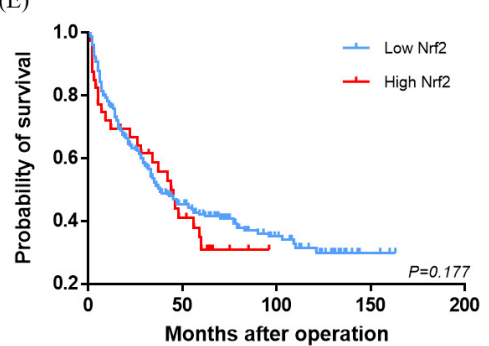

(C)

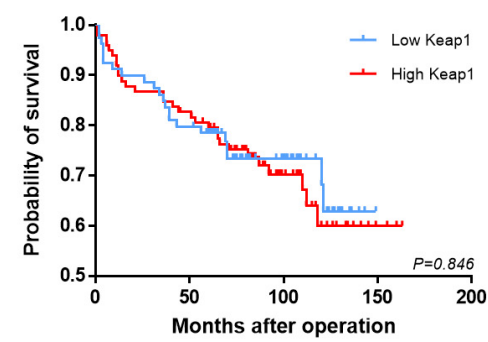

(F)

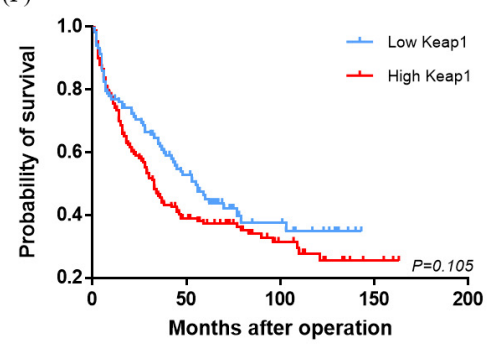

Figure 3. Kaplan-Meier survival curves comparing the overall survival $(\mathbf{A}-\mathbf{C})$ and disease-free survival (D-F) of hepatocellular carcinomas according to pNrf2 (A,D), Nrf2 (B,E), and Keap1 (C,F) expression status.

\subsection{Cytoplasmic Nrf2 Expression in HCCs and Clinicopathological Correlation}

Cytoplasmic Nrf2 expression was observed in 48/285 (16.8\%) of HCCs. There was no correlation between pNrf2 and Nrf2 expression status. Nrf2-high HCCs were smaller in size compared with Nrf2-low HCCs $(3.1 \pm 1.8 \mathrm{~cm}$ vs. $4.4 \pm 2.9 \mathrm{~cm}$, respectively, $p=0.005)$. Cytoplasmic Nrf2 expression was associated with increased expression of CAIX (20/48 (41.7\%) vs. 55/229 (24.0\%), respectively, $p=0.019)$ and Ezrin (31/48 (64.6\%) vs. 90/236 (38.1\%), respectively, $p=0.001)$ compared to Nrf2-low HCCs. Notably, Nrf2-high status was significantly associated with decreased OS in HCC patients on univariate analysis (HR, 3.33; 95\% CI, 1.74-6.37; $p<0.001$ ) (Table 3, Figure 3).

\subsection{Keap1 Expression in HCCs and Clinicopathological Correlation}

Keap1 was expressed in the cytoplasm of 161/285 (56.5\%) HCCs. Interestingly, a significant positive correlation was observed between $\mathrm{pNrf} 2$ and Keap1 expression $(p=0.020)$. Keap1 expression was associated with increased expression of uPAR (29/124 (23.4\%) vs. 55/159 (34.6\%), respectively, $p=0.049)$. In addition, more frequent p53 expression (47/147 (32.0\%) vs. 19/118 (16.1\%), respectively, $p=0.004$ ) and higher proliferative activity (Ki-67 labeling index: $8.1 \pm 10.2 \%$ vs. $5.7 \pm 8.5 \%$, respectively, $p=0.035$; mitotic index: $13.1 \pm 16.9 / 10 \mathrm{HPF}$ vs. $10.5 \pm 13.6 / 10 \mathrm{HPF}$, respectively, $p=0.056$ ) were noted in Keap1-high HCCs, compared to Keap1-low HCCs.

The positive correlation between pNrf2 and Keap1 and the association between Keap1 and increased proliferative activity in this study were unexpected, as Keap1 is known to suppress Nrf2 activation and, thus, expected to have tumor-suppressive effects. We, thus, analyzed the clinicopathological features of Keap1-positive HCCs separately in CAIX-positive $(n=75)$ and CAIX-negative HCCs $(n=202)$. Of note, we found that Keap1 expression was associated with increased Ki-67 labeling index $(7.4 \pm 10.3 \%$ vs. $4.6 \pm 7.1 \%$, respectively, $p=0.008)$, more frequent portal vein invasion $(1 / 202(0.9 \%)$ vs. $6 / 75(13.6 \%)$, respectively, $p=0.024)$, ezrin expression $(3 / 91(3.3 \%)$ vs. $13 / 110(11.8 \%)$, respectively, $p=0.035)$, uPAR expression $(15 / 91(16.5 \%)$ vs. $33 / 110(30.0 \%)$, respectively, $p=0.031)$, and p53 expression $(31 / 202(29.8 \%)$ vs. $14 / 75(34.1 \%)$, respectively, $p=0.009)$ only in CAIX-negative HCCs, while there was no association between Keap1 status and the clinicopathological factors in CAIX-positive HCCs (Table S1). In addition, although there were no differences in patient survival according to Keap1 expression status, there was interestingly a tendency for decreased DFS for Keap1-high HCCs in the CAIX-negative HCC group $(p=0.062)$. 


\subsection{Multivariate Survival Analysis}

Multivariate survival analysis was performed with clinicopathological and immunohistochemical parameters that demonstrated statistical significance on univariate analysis, including pNrf2 and cytoplasmic Nrf2. Other parameters associated with decreased OS on univariate analysis included tumor multiplicity (HR, 2.81; 95\% CI, 1.49-5.31; $p=0.001)$, tumor size ( $\geq 5 \mathrm{~cm}$ vs. $<5 \mathrm{~cm}$ ) (HR, 2.40; 95\% CI, 1.32-4.38; $p=0.004)$, portal vein invasion (HR, 3.29;95\% CI, 1.39-7.80; $p=0.007)$, the presence of extrahepatic metastasis (HR, 4.46; 95\% CI, 2.45-8.13; $p<0.001$ ), and expression of CAIX (HR, $1.85 ; 95 \%$ CI, 1.02-3.39; $p=0.044)$ and ezrin (HR, 3.16; 95\% CI, 1.67-5.97; $p<0.001)$. Multivariate analysis revealed cytoplasmic Nrf2 expression to be a significant independent prognostic factor for OS (HR, $4.35 ; 95 \%$ CI, 2.11-8.97; $p<0.001$ ), along with tumor multiplicity (HR, 1.98;95\% CI, 1.02-3.86; $p=0.045$ ), presence of extrahepatic metastasis (HR, 3.75; 95\% CI, 1.91-7.36; $p<0.001)$, and ezrin expression (HR, 2.41; 95\% CI, $1.25-4.64 ; p=0.009$ ) (Table 3). As for DFS, tumor multiplicity (HR, $1.49 ; 95 \%$ CI, $1.02-2.18 ; p=0.042)$ and the presence of extrahepatic metastasis (HR, 4.48; 95\% CI, 3.15-6.35; $p<0.001$ ) were the only significant independent predictors on multivariate analysis, and none of the immunohistochemical markers were associated with poor DFS.

\section{Discussion}

In this study, we examined the expression of Nrf2, pNrf2, and Keap1 in HCCs, and we correlated the findings with the clinicopathological parameters and expression status of stemness, hypoxia, and EMT-related markers. The association between Nrf2 expression and poor prognosis was previously demonstrated in various solid malignancies, including in two meta-analysis studies [18,21]; however, the prognostic value of Nrf2/Keap1 in HCCs was only reported in two studies so far [16,22]. Zhang et al. demonstrated an association between Nrf2 expression in HCCs and decreased survival, poor histological differentiation, increased proliferation, and more frequent metastasis [22]. Although we observed a significant association between strong cytoplasmic Nrf2 expression in HCC with decreased OS and EMT-related marker expression, other clinicopathological factors such as proliferative activity were not significantly influenced by Nrf2 expression status. The different antibodies used may partly explain the differences; Zhang et al. described nucleocytoplasmic Nrf2 expression with predominantly nuclear localization in their study, while Nrf2 expression in the current study was cytoplasmic. As it is the nuclear form of Nrf2 that is recognized to be the active pro-tumorigenic form, we also examined nuclear pNrf2 expression in HCC, and we found that pNrf2-high HCCs were associated with poor prognosis, increased proliferative activity, and more frequent expression of markers associated with stemness, EMT, and hypoxia. The association between pNrf2 expression and decreased survival in HCCs is in line with a previous study by Chen et al., although we did not find the same expected positive correlation between pNrf2 and Nrf2 expression or an inverse correlation between pNrf2 and Keap1 expression that they observed [16]. It should be noted that Chen et al. reported a larger proportion of pNrf2-positive HCCs (50\%) which could be explained by the different cut-offs; we evaluated pNrf2 using a quantitative approach and defined "pNrf2-high" HCCs $(\sim 25 \%)$ as those with strong nuclear labeling exceeding $10 \%$, while the former study adopted a semiquantitative approach for both $\mathrm{pNrf} 2$ and Keap1, counting any labeling for $\mathrm{pNrf} 2$ and Keap1 (in the nucleus and cytoplasm, respectively) that was more than focal weak positive as "positive" [16]. Nevertheless, despite the differences in interpretation methods, it is interesting that pNrf2 expression was associated with CAIX, EpCAM, K19, and ezrin expression in this study.

A subset of HCCs that are morphologically compatible with HCC but express immunohistochemical markers associated with stemness (e.g., K19, EpCAM, and SAL-like protein 4 (SALL4)) recently received increasing attention, as these HCCs demonstrate more aggressive behavior compared to conventional HCCs, expression of EMT-related markers, poor prognosis, and resistance to locoregional and systemic treatment [23-25]. HCCs expressing stemness-related markers were shown to more frequently result in residual tumors after transarterial chemoembolization (TACE), compared to conventional HCCs, and the residual tumors after TACE often expressed stemness-related markers 
that were co-localized with CAIX expression, thus suggesting a relationship between TACE-induced hypoxia and stemness [26]. In this study, we found positive correlations between $\mathrm{pNrf2}$ expression and hypoxia, stemness, and EMT-related markers, suggesting a possible role for Nrf2 in the link between tumor hypoxia, stemness, and aggressive behavior of HCC. pNrf2 may be upregulated in HCCs in response to oxidative stress, and pNrf2 may further enhance the aggressive behavior of hypoxic HCCs; however, functional studies would be necessary for validation.

Although Keap1 expression status was not correlated with clinicopathological factors or patient survival, Keap1-high HCCs more frequently demonstrated p53 expression and higher proliferative activity. In addition, a positive correlation was found between Keap1 and pNrf2 expression status. These results are somewhat counterintuitive, as Keap1 was described to be a protein that suppresses Nrf2 activation. Interestingly, when we examined the transcriptomics data provided by The Human Protein Atlas (https://www.proteinatlas.org/) and UALCAN (http://ualcan.path.uab.edu/), which are based on The Cancer Genome Atlas (TCGA) data, we found that KEAP1 messenger RNA (mRNA) expression was in fact higher in HCCs than in non-neoplastic liver tissues, and high KEAP1 mRNA was associated with poor prognosis in HCCs (Figure S2) [27,28]. An association between Keap1 expression and poor prognosis was also demonstrated in endometrial cancers, and the authors postulated that the overexpression of Keap1 may reflect Keap1 induction in the setting of oxidative stress [15]. On the other hand, when we analyzed the Keap1 expression status separately in CAIX-positive and CAIX-negative HCCs, we interestingly found that Keap1 expression was associated with more frequent portal vein invasion, higher proliferative activity, frequent p53 expression, and a tendency for decreased DFS in CAIX-negative HCCs, while Keap1 was not associated with any of the clinicopathological or immunohistochemical factors in the CAIX-positive HCCs. Instead, Nrf2 and/or pNrf2 were the determinants of aggressive behavior and poor survival in the setting of CAIX-positive HCCs. Thus, it is possible that, under hypoxic stress, Nrf2 is released from Keap1 to contribute to the aggressive behavior of HCC, while, under normoxic conditions, Keap1 is also a determinant of aggressive behavior. In addition, the association between strong cytoplasmic Nrf2 expression and decreased OS in this study suggests that, although the nuclear $\mathrm{pNrf2}$ is the active form of $\mathrm{Nrf2}$, strong overexpression of Nrf2 in the cytoplasm is also abnormal and has prognostic implications.

There are a few limitations to this study. Firstly, although we analyzed a large number of HCCs, this is a retrospective cohort study using formalin-fixed paraffin-embedded tissue samples and the results are based on immunohistochemical results. As all the HCCs enrolled in this study were surgically resected HCCs, it is possible that early well-differentiated HCCs-which are often treated by loco-regional modalities-were less likely to be included in this study, resulting in a biased cohort predominantly consisting of moderately to poorly differentiated HCCs. Functional studies would need to be performed to further evaluate the functional relationships among Nrf2/Keap1, hypoxia, and EMT. Secondly, as the majority of the HCCs in this study have an HBV-related etiology, it would be necessary to perform a similar analysis on an independent cohort of HCCs from regions where HBV is not as predominant.

\section{Materials and Methods}

\subsection{Patients}

A total of 285 cases of primary HCCs that were surgically resected between 2003 and 2012 at Seoul National University Bundang Hospital (Seongnam, Korea) were evaluated in this study. Clinicopathological information was obtained from the patients' electronic medical records, pathology database, and a review of the archived glass slides, and it included sex, age at operation, underlying etiology (including hepatitis B virus (HBV), hepatitis $\mathrm{C}$ virus (HCV), alcohol and non-alcoholic fatty liver disease (NAFLD)), tumor size, multiplicity, tumor differentiation (Edmonson-Steiner grade), presence of microvascular and portal vein invasion, mitotic index, extrahepatic metastasis, and pathological $\mathrm{T}$ stages according to the American Joint Committee on Cancer TNM staging system (eighth edition). 
The mitotic index was expressed as the total number of mitotic figures in 10 high-power fields (HPFs). The clinical follow-up information, including the dates of surgery, disease recurrence, death, and most recent follow-up, was retrieved from the electronic medical records. Overall survival (OS) was defined as the number of months between surgery and patient death, and disease-free survival (DFS) was defined as the number of months between surgery and disease recurrence (local recurrence and/or distant metastasis). In addition, a separate independent tissue microarray cohort of 293 resected HCCs from Seoul National University Hospital was evaluated to further examine the prognostic significance of pNrf2 expression. This study was approved by the Institutional Review Board of Seoul National University Hospital and Seoul National University Bundang Hospital (joint IRB\# H-1808-073-965), and patient consent was waived due to the retrospective nature of this study.

\subsection{Tissue Samples and Immunohistochemistry}

Tissue microarrays with tissue cores measuring $2 \mathrm{~mm}$ in diameter were constructed from formalin-fixed paraffin-embedded HCC tissue, and they were arranged in recipient tissue microarray blocks using a trephine apparatus (Superbiochips Laboratories, Seoul, Korea). Immunohistochemistry was performed on 4- $\mu$ m-thick tissue sections using antibodies for pNrf2, Nrf2, Keap1, K19, EpCAM, E-cadherin, CAIX, p53, and Ki-67, using an automated platform (Ventana BenchMark GX, Ventana Medical Systems, Oro Valley, AZ, USA). Immunohistochemical staining for ezrin and uPAR was performed manually, with antigen retrieval using the citrate buffer ( $\mathrm{pH}$ 6.0) and the Envision Kit (Dako, Glostrup, Denmark). Detailed information on the antibodies is summarized in Table 4.

Table 4. Summary of the antibodies used in this study.

\begin{tabular}{|c|c|c|c|c|}
\hline Antibody & Description & Source & Dilution & Method \\
\hline \multirow{2}{*}{ Nrf2 } & \multirow{2}{*}{ Rabbit monoclonal (EP1808Y) } & Abcam & \multirow{2}{*}{$1: 50$} & \multirow{2}{*}{ Autostainer } \\
\hline & & (Cambridge, UK) & & \\
\hline \multirow{2}{*}{ pNrf2 } & \multirow{2}{*}{ Rabbit monoclonal (EP1809Y) } & Abcam & \multirow{2}{*}{$1: 100$} & \multirow{2}{*}{ Autostainer } \\
\hline & & (Cambridge, UK) & & \\
\hline \multirow{2}{*}{ Keap1 } & \multirow{2}{*}{ Rabbit polyclonal } & Proteintech & \multirow{2}{*}{$1: 300$} & \multirow{2}{*}{ Autostainer } \\
\hline & & (Manchester, UK) & & \\
\hline \multirow{2}{*}{ Keratin 19 (K19) } & \multirow{2}{*}{ Mouse monoclonal (RCK108) } & Dako & \multirow{2}{*}{$1: 200$} & \multirow{2}{*}{ Autostainer } \\
\hline & & (Glostrup, Denmark) & & \\
\hline \multirow{2}{*}{ EpCAM } & \multirow{2}{*}{ Mouse monoclonal (VU-1D9) } & Millipore & \multirow{2}{*}{$1: 1500$} & \multirow{2}{*}{ Autostainer } \\
\hline & & (Temecula, CA, USA) & & \\
\hline \multirow{2}{*}{ Ezrin } & \multirow{2}{*}{ Mouse monoclonal (3C12) } & Abcam & \multirow{2}{*}{$1: 100$} & \multirow{2}{*}{ Manual (pH 6.0) } \\
\hline & & (Cambridge, UK) & & \\
\hline \multirow{2}{*}{ uPAR } & \multirow{2}{*}{ Mouse monoclonal (R-4) } & Abcam & \multirow{2}{*}{$1: 40$} & \multirow{2}{*}{ Manual (pH 6.0) } \\
\hline & & (Cambridge, UK) & & \\
\hline \multirow{2}{*}{ E-cadherin } & \multirow{2}{*}{ Mouse monoclonal (36B5) } & Novocastra & \multirow{2}{*}{$1: 100$} & \multirow{2}{*}{ Autostainer } \\
\hline & & (Newcastle, UK) & & \\
\hline \multirow{2}{*}{ CAIX } & \multirow{2}{*}{ Rabbit polyclonal } & Abcam & \multirow{2}{*}{$1: 500$} & \\
\hline & & (Cambridge, UK) & & Autostainer \\
\hline 053 & Mouse monoclonal (DO-7) & Dako & & \\
\hline p53 & Mouse monoclonal (DU-7) & (Glostrup, Denmark) & 1:1000 & Autostainer \\
\hline 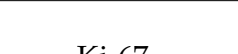 & Mour monolonol(MID 1) & Dako & 1,100 & $A=$ \\
\hline $\mathrm{K} 1-6 /$ & 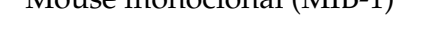 & (Glostrup, Denmark) & $1: 100$ & Autostainer \\
\hline
\end{tabular}


For Nrf2 and Keap1, the intensity of cytoplasmic expression in the tumor cells was recorded as 0 (negative), $1+$ (weak), $2+$ (moderate), and 3+ (strong). Nrf2 expression was cytoplasmic, and strong positive (3+) staining was designated as "Nrf2-high" (overexpression). "Keap1-high" was defined as moderate to strong cytoplasmic staining in the tumor cells, and the staining intensity was similar to or more intense than that seen in non-neoplastic hepatocytes, cholangiocytes, and stromal cells. pNrf2 staining was evaluated in a quantitative manner, using the ImageJ software (National Institutes of Health, Bethesda, MD, USA); the percentage of nuclear pNrf2 labeling was calculated by dividing the number of positively stained tumor cell nuclei by the total number of tumor cell nuclei, in an HPF (400× magnification). "pNrf2-high" was defined as pNrf2 labeling in $\geq 10 \%$ of tumor cells. K19, EpCAM, CAIX, ezrin, and uPAR expression was designated positive when there was cytoplasmic (K19, CAIX, ezrin) or membranous (EpCAM and uPAR) expression in $\geq 5 \%$ of the tumor cells. Loss of E-cadherin expression was defined as complete absence of membranous E-cadherin expression in the tumor cells. p53 expression was defined as positive when strong nuclear labeling was present in more than $10 \%$ HCC cells. Two pathologists (K.L. and H.K.) independently evaluated the immunohistochemical stains and reached an agreement using a multihead microscope when there were discrepancies in the interpretation results.

\subsection{Statistical Analysis}

All data were analyzed using IBM SPSS statistics software version 25.0 (SPSS Inc., Chicago, IL, USA) and GraphPad Prism software version 7.0 (San Diego, CA, USA), and a $p$-value $<0.05$ was considered statistically significant. The associations between the immunohistochemical stain results and clinicopathological parameters were assessed using the $\chi^{2}$ test, Fisher exact test, and Student's $t$-test, as deemed appropriate. OS and DFS were analyzed using the Kaplan-Meier method and the log-rank test. Multivariate analysis using the Cox regression model was performed for clinicopathological and immunohistochemical parameters with $p<0.05$ on univariate analysis.

\section{Conclusions}

In summary, we demonstrate that pNrf2 expression in HCC was associated with increased proliferative activity, decreased survival, and more frequent expression of hypoxia, stemness, and EMT-related markers, suggesting a possible role for $\mathrm{pNrf2}$ as a marker of aggressive behavior and poor prognosis in HCC. In addition, contrary to previous knowledge, strong cytoplasmic Nrf2 expression was also an independent predictor of decreased survival in HCC, and Keap1 expression was associated with aggressive HCC behavior in CAIX-negative tumors, suggesting that Keap1 expression in HCCs should be interpreted in the context of the metabolic status. Further prognostic studies in independent cohorts and functional studies would be required to validate the current findings.

Supplementary Materials: The following are available online at http://www.mdpi.com/2072-6694/12/8/2128/s1. Figure S1: Kaplan-Meier survival curves demonstrating decreased overall survival (A) and disease-free survival (B) in pNrf2-high HCCs in an independent cohort of HCC $(n=293)$, Figure S2: Increased KEAP1 mRNA expression in HCCs compared to non-neoplastic liver tissue (A) and decreased overall survival in KEAP1-high HCCs (B) in the LIHC cohort from The Cancer Genome Atlas (graphs generated from UALCAN, http://ualcan.path.uab.edu/); Table S1. Clinicopathologic features according to Keap1 expression status in CAIX-positive and CAIX-negative hepatocellular carcinomas.

Author Contributions: Writing—original draft, K.L. (Kiryang Lee) and H.K.; writing—review and editing, K.L. (Kiryang Lee) and H.K.; conceptualization, K.L. (Kiryang Lee) and H.K.; resources, K.L. (Kiryang Lee), S.K., H.K., S.J.Y., Y.L. (Yangkyu Lee), H.L., Y.L. (Youngeun Lee), H.P., J.H.N., S.A., and K.L. (Kyoungbun Lee); formal analysis, K.L. (Kiryang Lee), S.K., and H.K.; software, K.L. (Kiryang Lee) and S.K.; visualization, K.L. (Kiryang Lee); supervision, H.K.; funding acquisition, H.K. All authors have read and agreed to the published version of the manuscript.

Funding: This research was funded by National Research Foundation of Korea (NRF) grant funded by the Korea government (MSIT) (NRF-2019R1A2C2010056, NRF-2016R1D1A1A09919042).

Conflicts of Interest: The authors declare no conflicts of interest. 


\section{References}

1. Ferlay, J.; Colombet, M.; Soerjomataram, I.; Mathers, C.; Parkin, D.M.; Piñeros, M.; Znaor, A.; Bray, F. Estimating the global cancer incidence and mortality in 2018: GLOBOCAN sources and methods. Int. J. Cancer 2019, 144, 1941-1953. [CrossRef] [PubMed]

2. Fujimoto, A.; Furuta, M.; Totoki, Y.; Tsunoda, T.; Kato, M.; Shiraishi, Y.; Tanaka, H.; Taniguchi, H.; Kawakami, Y.; Ueno, M.; et al. Whole-genome mutational landscape and characterization of noncoding and structural mutations in liver cancer. Nat. Genet. 2016, 48, 500-509. [CrossRef] [PubMed]

3. Guichard, C.; Amaddeo, G.; Imbeaud, S.; Ladeiro, Y.; Pelletier, L.; Ben Maad, I.; Calderaro, J.; Bioulac-Sage, P.; Letexier, M.; Degos, F; et al. Integrated analysis of somatic mutations and focal copy-number changes identifies key genes and pathways in hepatocellular carcinoma. Nat. Genet. 2012, 44, 694. [CrossRef] [PubMed]

4. Cancer Genome Atlas Research Network. Comprehensive and Integrative Genomic Characterization of Hepatocellular Carcinoma. Cell 2017, 169, 1327-1341. [CrossRef] [PubMed]

5. Torbenson, M.S.; Ng, I.O.L.; Park, Y.N.; Roncalli, M.; Sakamoto, M. Hepatocellular carcinoma. In WHO Classification of Tumours Editorial Board, 5th ed.; Digestive System Tumours, Ed.; International Agency for Research on Cancer: Lyon, France, 2019; pp. 229-239.

6. Zucman-Rossi, J.; Villanueva, A.; Nault, J.C.; Llovet, J.M. Genetic Landscape and Biomarkers of Hepatocellular Carcinoma. Gastroenterology 2015, 149, 1226-1239. [CrossRef]

7. Sporn, M.B.; Liby, K.T. NRF2 and cancer: The good, the bad and the importance of context. Nat. Rev. Cancer 2012, 12, 564-571. [CrossRef]

8. Suzuki, T.; Motohashi, H.; Yamamoto, M. Toward clinical application of the Keap1-Nrf2 pathway. Trends Pharm. Sci. 2013, 34, 340-346. [CrossRef]

9. Chun, Y.; Ye-xiong, T.; Guang-zhen, Y.; Jian, Z.; Yu-fei, P.; Chen, L.; Jing, F.; Yao, C.; Zhi-wen, D.; Li-wei, D.; et al. Gankyrin has an antioxidative role through the feedback regulation of Nrf2 in hepatocellular carcinoma. J. Exp. Med. 2016, 213, 859-875.

10. Kahroba, H.; Shirmohamadi, M.; Hejazi, M.S.; Samadi, N. The Role of Nrf2 signaling in cancer stem cells: From stemness and self-renewal to tumorigenesis and chemoresistance. Life Sci. 2019, 239, 116986. [CrossRef]

11. Jaramillo, M.C.; Zhang, D.D. The emerging role of the Nrf2-Keap1 signaling pathway in cancer. Genes Dev. 2013, 27, 2179-2191. [CrossRef]

12. Ngo, H.K.C.; Kim, D.H.; Cha, Y.N.; Na, H.K.; Surh, Y.J. Nrf2 Mutagenic Activation Drives Hepatocarcinogenesis. Cancer Res. 2017, 77, 4797-4808. [CrossRef] [PubMed]

13. Raghunath, A.; Sundarraj, K.; Arfuso, F.; Sethi, G.; Perumal, E. Dysregulation of Nrf2 in Hepatocellular Carcinoma: Role in Cancer Progression and Chemoresistance. Cancers 2018, 10, 481. [CrossRef] [PubMed]

14. Li, C.; Cheng, L.; Wu, H.; He, P.; Zhang, Y.; Yang, Y.; Chen, J.; Chen, M. Activation of the KEAP1-NRF2-ARE signaling pathway reduces oxidative stress in Hep2 cells. Mol. Med. Rep. 2018, 18, 2541-2550. [CrossRef] [PubMed]

15. Ahtikoski, A.M.; Kangas, J.; Salonen, R.; Puistola, U.; Karihtala, P. Cytoplasmic Keap1 Expression Is Associated With Poor Prognosis in Endometrial Cancer. Anticancer. Res. 2019, 39, 585-590. [CrossRef]

16. Chen, J.; Yu, Y.; Ji, T.; Ma, R.; Chen, M.; Li, G.; Li, F.; Ding, Q.; Kang, Q.; Huang, D.; et al. Clinical implication of Keap1 and phosphorylated Nrf2 expression in hepatocellular carcinoma. Cancer Med. 2016, 5, $2678-2687$. [CrossRef]

17. Cho, H.Y.; Kim, K.; Kim, Y.B.; Kim, H.; No, J.H. Expression Patterns of Nrf2 and Keap1 in Ovarian Cancer Cells and their Prognostic Role in Disease Recurrence and Patient Survival. Int. J. Gynecol. Cancer 2017, 27, 412-419. [CrossRef]

18. Guo, Y.; Shen, L. Overexpression of NRF 2 is correlated with prognoses of patients with malignancies: A meta-analysis. Thorac. Cancer 2017, 8, 558-564. [CrossRef]

19. Isohookana, J.; Haapasaari, K.M.; Soini, Y.; Karihtala, P. Keap1 expression has independent prognostic value in pancreatic adenocarcinomas. Diagn. Pathol. 2015, 10, 28. [CrossRef]

20. Lingzhi, P.; Yaoxing, D.; Yongbin, Z.; Diande, Z.; Yanghui, W.; Jibin, Y.; Mingxu, D. Expression of Nrf2 and NQO1 in human gastric cancer and their clinical significance. Int. J. Clin. Exp. Pathol. 2016, 9, 1635-1643.

21. Wang, L.; Zhang, C.; Qin, L.; Xu, J.; Li, X.; Wang, W.; Kong, L.; Zhou, T.; Li, X. The prognostic value of NRF2 in solid tumor patients: A meta-analysis. Oncotarget 2018, 9, 1257-1265. [CrossRef] 
22. Zhang, M.; Zhang, C.; Zhang, L.; Yang, Q.; Zhou, S.; Wen, Q.; Wang, J. Nrf2 is a potential prognostic marker and promotes proliferation and invasion in human hepatocellular carcinoma. BMC Cancer 2015, $15,531$. [CrossRef] [PubMed]

23. Olivier, G.; Mina, K.; Johannes, B.; Bart, S.; Carl, J.; Francesca, L.; Aezam, K.; Jasper, W.; Léon, K.; Anne, D.; et al. Keratin 19: A key role player in the invasion of human hepatocellular carcinomas. Gut 2014, 63, 674-685.

24. Kawai, T.; Yasuchika, K.; Ishii, T.; Katayama, H.; Yoshitoshi, E.Y.; Ogiso, S.; Kita, S.; Yasuda, K.; Fukumitsu, K.; Mizumoto, M.; et al. Keratin 19, a Cancer Stem Cell Marker in Human Hepatocellular Carcinoma. Clin. Cancer Res. 2015, 21, 3081-3091. [CrossRef] [PubMed]

25. Kim, H.; Choi, G.H.; Na, D.C.; Ahn, E.Y.; Kim, G.I.; Lee, J.E.; Cho, J.Y.; Yoo, J.E.; Choi, J.S.; Park, Y.N. Human hepatocellular carcinomas with "Stemness"-related marker expression: Keratin 19 expression and a poor prognosis. Hepatology 2011, 54, 1707-1717. [CrossRef] [PubMed]

26. Rhee, H.; Nahm, J.H.; Kim, H.; Choi, G.H.; Yoo, J.E.; Lee, H.S.; Koh, M.J.; Park, Y.N. Poor outcome of hepatocellular carcinoma with stemness marker under hypoxia: Resistance to transarterial chemoembolization. Mod. Pathol. 2016, 29, 1038-1049. [CrossRef]

27. Uhlen, M.; Fagerberg, L.; Hallstrom, B.M.; Lindskog, C.; Oksvold, P.; Mardinoglu, A.; Sivertsson, A.; Kampf, C.; Sjöstedt, E.; Asplund, A.; et al. Proteomics. Tissue-based map of the human proteome. Science 2015, 347, 1260419. [CrossRef]

28. Chandrashekar, D.S.; Bashel, B.; Balasubramanya, S.A.H.; Creighton, C.J.; Ponce-Rodriguez, I.; Chakravarthi, B.V.; Varambally, S. UALCAN: A Portal for Facilitating Tumor Subgroup Gene Expression and Survival Analyses. Neoplasia 2017, 19, 649-658. [CrossRef]

(C) 2020 by the authors. Licensee MDPI, Basel, Switzerland. This article is an open access article distributed under the terms and conditions of the Creative Commons Attribution (CC BY) license (http://creativecommons.org/licenses/by/4.0/). 